\title{
Simultaneous HPLC Assay of Paracetamol and Sulfapyridine as Markers for Estimating Gastrointestinal Transit of Amphotericin B-containing Nanoparticles in Rat Plasma
}

\author{
Hilda $\mathrm{A}^{1}$, Nashiru $\mathrm{B}^{* 1}$ and Kah-Hay $\mathrm{Y}^{2}$
}

${ }^{1}$ School of Pharmacy, University of Nottingham, Malaysia Campus, 43500 Selangor, Malaysia

${ }^{2}$ School of Pharmacy, University of Science, Malaysia 11800, Minden, Penang Malaysia

*Corresponding author: Nashiru B, School of Pharmacy, University of Nottingham, Malaysia Campus, 43500 Selangor, Malaysia, Fax: +60389248018, Tel: +6038924821, Email: Nashiru.Billa@nottingham.edu.my Citation: Hilda A, Nashiru B, Kah-Hay Y (2015) Simultaneous HPLC Assay of Paracetamol and Sulfapyridine as Markers for Estimating Gastrointestinal Transit of Amphotericin B-containing Nanoparticles in Rat Plasma. J Bioequiv 1(1): 104. doi: 10.15744/2575-551X.1.104

Received Date: May 18, 2015 Accepted Date: September 02, 2015 Published Date: September 04, 2015

\begin{abstract}
A simple and sensitive two-step reversed-phase HPLC method was developed and validated for determining amphotericin B, paracetamol and sulfapyridine in rat plasma using piroxicam as internal standard. This was subsequently employed in a pharmacokinetic study in rats following simultaneous oral administration of solid lipid nanoparticle formulations of amphotericin B, paracetamol and sulfasalazine. The paracetamol and sulfapyridine served as markers for estimating gastric emptying and cecal arrival times. A gradient elution was employed in the analysis and detection at $405 \mathrm{~nm}$ for amphotericin B and $254 \mathrm{~nm}$ for paracetamol and sulfapyridine. The internal standard was detectable at both wavelengths. The gastrointestinal study was conducted on male Sprague-Dawley rats. Calibration curves were linear for the three drugs $\left(r^{2} \geq 0.9998\right)$ with the detection and quantification limits comparable with those in literature. Drug recovery from rat plasma was more than $92 \%$. The accuracy of the method was $<8 \%$ for the three drugs whilst the precision was $10 \%$. Paracetamol was rapidly absorbed from the small intestine reaching its $\mathrm{C}_{\max }$ in $1 \mathrm{hr}$ while amphotericin $\mathrm{B}$ was absorbed slowly reaching peak levels in $8 \mathrm{hr}$. Sulfapyridine was absorbed only after a lag time of $2 \mathrm{hr}$ due to delayed arrival and hydrolysis of sulfasalazine at the colon by azo-reductase bacteria. The HPLC method is rapid and applicable for the simultaneous estimation amphotericin $B$, paracetamol and sulfapyridine in rat plasma, which may serve for an indirect estimation of gastric emptying and orocecal transit properties of amphotericin B within dosage forms after oral administration.
\end{abstract}

Keywords: Amphotericin B; Paracetamol; Sulfapyridine; Sulfasalazine; Gastrointestinal transit

\section{Introduction}

Amphotericin B (AmB) is a broad-spectrum antifungal drug chiefly used for managing life-threatening systemic fungal infections. A micellar suspension of the sodium deoxycholate salt of amphotericin B (Fungizone ${ }^{\circledast}$ ) is widely used currently as the conventional option. However, three other lipid-based formulations are available but used to a lesser extent: AmB lipid complex (AmB complexed with dimyristoylphosphatidylcholine and dimyristoylphosphatidylglycerol, Abelcet ${ }^{\circledR}$ ); AmB colloidal dispersion (AmB complexed with cholesteryl sulfate, Amphocil ${ }^{\circledR}$ ) and liposomal AmB (AmB complexed with hydrogenated soy phosphatidylcholine, distearoyl, phosphatidylglycerol and cholesterol, AmBisome $\left.{ }^{\circledR}\right)[1,2]$.

The mode of administration of AmB in these formulations is limited to intravenous injection due to its poor aqueous solubility, which makes systemic absorption from the gastrointestinal (GI) tract negligible. Thus, research has intensified in formulating AmB into a suitable oral preparation that addresses the above constraint since intravenous administration of is associated with several adverse effects such as fever, chills, rigors, malaise, headache, generalized aches, nausea, vomiting and hypoxia [3]. These adverse reactions are partly related to the induction of pro-inflammatory cytokines produced by AmB and also the release of TNF-alpha from macrophages $[4,5]$.

Solid lipid nanoparticles (SLN) can be formulated to incorporate hydrophobic drugs such as AmB [6-10]. Due to their hydrophobicity and relatively high surface area to volume ratio, SLN can be effectively taken up by the Peyer's patches within the GI tract which improves drug bioavailability [11]. Furthermore, SLN exhibit mucoadhesion to the gut wall which therefore creates a desirable platform for the establishment of a concentration gradient with blood and further enhances drug absorption [12,13].

Data on the transit behavior of dosage forms through the GI tract is essential in determining possible absorption windows of the drug payload. This information can in turn be used to optimize desirable properties from the dosage form with a view to improving drug bioavailability. 
The GI transit properties of dosage forms can be studied using gamma scintigraphy [14] and nuclear magnetic resonance [15]. An indirect means of studying GI transit properties of dosage forms using marker drugs is less costly and has been used successfully by several researchers [16-18]. The indirect approach is based on the premise that certain drugs have preferential absorption at specific locations within the GI tract. For example, following oral administration, the absorption of paracetamol (PAR) which is preferential in the small intestine, is related to the rate of gastric emptying of adosage form containing it [19]. On the other hand, when sulfasalazine (SSZ) is administered orally, it is hydrolyzed by the large bowel flora to produce sulfapyridine (SP) and 5 -aminosalicylic acid. The rate of appearance of absorbed SP in the blood can also be used to estimate the orocecal transit time $[20,21]$ of a SSZ-loaded dosage form. Therefore, these two drugs can be used as markers to estimate the gastric emptying and orocecal transit times [16-18] of AmB-containing SLN.

A crucial aspect of utilizing the indirect GI transit estimation of dosage forms based on marker drugs is that the analytical method must be validated and be sufficiently sensitive to detect minute concentrations of the marker drugs. A further desirable requirement is the simplicity of the method, given that several drugs would have to be analyzed. In this paper, we propose a simple and sensitive HPLC method for the simultaneous assay of AmB, PAR and SP in rat plasma. This method was later used in a GI transit study to determine the GI transit and pharmacokinetic data of AmB following oral administration of AmB SLN as well as the marker drugs, PAR SLN and SSZ SLN [with SP to be released from SSZ in-vivo] using rats as the animal model.

\section{Experimental}

\section{Chemicals and reagents}

Acetonitrile was purchased from Duksan Pure Chemicals Co. Ltd. (Ansan, Korea). Paracetamol, dimethyl sulfoxide (DMSO) and sulfapyridine were purchased from Sigma-Aldrich (Sigma-Aldrich Co. LLC., St. Louis, USA) and amphotericin B from Nacalai Tesque (Kyoto, Japan). Methanol and ethanol were purchased from Fisher Scientific (Waltham, USA). Water was Milli-Q 18.2 $\mathrm{M} \Omega . \mathrm{cm}$ at $25^{\circ} \mathrm{C}$ (Millipore Corp., Bedford, USA). Acetic acid and sodium acetate trihydrate were purchased from R \& M Chemicals (Essex, UK). All reagents and solvents used were of analytical and HPLC grades respectively.

\section{HPLC instrumentation and conditions}

A Perkin Elmer HPLC system (Series 200 auto-sampler, Series 200 UV/ VIS detector, Waltham, USA) configured to TotalChrom ${ }^{\circledR}$ Navigator software was used to analyze drug samples in the plasma. Separation of peaks was achieved using an Eclipse Plus ${ }^{\circledR}$ C18 $(250 \times 4.6 \mathrm{~mm}, 5 \mu \mathrm{m})$ column (ZORBAX, Agilent, USA). The mobile phase was filtered through a $0.45 \mu \mathrm{m}$ membrane filter before use and comprised of acetate buffer $(\mathrm{pH} 4)$, prepared by mixing $847 \mathrm{~mL}$ of $0.1 \mathrm{M}$ acetic acid and $153 \mathrm{~mL} 0.1 \mathrm{M}$ sodium acetate (trihydrate): [A] and acetonitrile: [B] and run in gradient mode as follows: $100 \% \mathrm{~A}$ at $0-0.5 \mathrm{~min} ; 60 \% \mathrm{~A}$ at $0.5-7 \mathrm{~min} ; 20 \% \mathrm{~A}$ at $7-9$ min; $60 \% \mathrm{~A}$ at $9-12 \mathrm{~min}$ and $100 \% \mathrm{~A}$ at $12-15 \mathrm{~min}$, at a flow rate of $1 \mathrm{~mL} / \mathrm{min}$. The HPLC was run in a two-step injection mode to detect PAR and SP at $254 \mathrm{~nm}$ and then AmB at $405 \mathrm{~nm}$. Piroxicam as internal standard (IS) was dissolved in a 1:1 methanol-ethanol solution to prepare a $5 \mu \mathrm{g} / \mathrm{mL}$ stock solution. The IS was detectable at both wavelengths. The methanol-ethanol mixture also served as deproteinizing solvent for the plasma samples.

\section{Plasma standards}

Stock plasma solutions containing AmB, PAR and SP at $100 \mu \mathrm{g} / \mathrm{mL}, 200 \mu \mathrm{g} / \mathrm{mL}$ and $1000 \mu \mathrm{g} / \mathrm{mL}$ respectively were prepared by spiking appropriate amounts of the respective drug solutions into the appropriate volumes of plasma. The stock solutions were further diluted with plasma to prepare the calibration standards for each drug, at three concentration levels representing low (LC), mid-range (MC) and high (HC) concentrations. The LC, MC and $\mathrm{HC}$ for AmB were 0.05, 0.25 and $10 \mu \mathrm{g} / \mathrm{mL}$; for PAR were 0.5, 2.5 and $100 \mu \mathrm{g} / \mathrm{mL}$; and for SP were $0.1,0.5$ and $20 \mu \mathrm{g} / \mathrm{mL}$.

\section{Plasma sample preparation}

Samples for drug analyses were prepared by transferring $100 \mu \mathrm{L}$ of plasma and $150 \mu \mathrm{L}$ of the IS solution into microcentrifuge tubes. The tubes were vortex-mixed for $5 \mathrm{~min}$ and the extracts centrifuged at 14,000 rpm for $10 \mathrm{~min}$. The clear supernatants were aspirated and filtered through a $0.20 \mu \mathrm{m}$ syringe filter followed by direct injection onto the HPLC system.

\section{Linearity}

Calibration curves for AmB, PAR and SP in drug spiked-plasma were obtained over the following concentration ranges; 0.05 - 10 $\mu \mathrm{g} / \mathrm{mL}, 0.5-100 \mu \mathrm{g} / \mathrm{mL}$ and $0.1-20 \mu \mathrm{g} / \mathrm{mL}$ respectively. The calibration curves were constructed using the ratio of the peak signal of each drug concentration to that of the internal standard versus corresponding drug concentration. Each analysis represents a replica of six runs.

\section{Precision and accuracy}

The within-day and between-day precision of the analysis at each concentration for each drug was determined as the percentage coefficient of variation $(\% \mathrm{CV})$ using the following relationship: 


$$
\% \mathrm{CV}=\frac{\text { Standard deviation (SD) }}{\text { Mean concentration }} \times 100 \%
$$

The mean represents a replica of six determinations.

The within-day and between-day accuracy of the analyses was determined as the relative error at each concentration for each drug, using the following relationship:

$$
\text { Relative error }\left(E_{r}\right)=\frac{\bar{x}-x_{t}}{x_{t}} \times 100 \% \ldots \ldots \ldots
$$

Where: $\bar{x}=$ measured value(average); $x_{t}=$ true value

\section{Recovery}

The recoveries of AmB, PAR and SP from spiked plasma following the extraction procedure were determined by comparing the peak signal of each drug from the prepared plasma samples with that of solutions of the pure drug samples in a solvent mix of DMSO and methanol. Six determinations were done for each drug, covering the three concentration levels explained in section 4.3. The percentage recovery was determined using the following relationship:

$$
\% \text { Recovery }=\frac{\text { Concentration obtained from spiked plasma }}{\text { Concentration of pure drug solutions }} \times 100 \% \ldots \ldots \ldots
$$

\section{Specificity and sensitivity}

The specificity of the analysis was performed by observing the chromatogram for blank plasma samples to detect any possible peak interference.

The sensitivity was determined in terms of limit of detection (LOD) and limit of quantization (LOQ), which were determined as the lowest concentration of each analyte at which the signal-to-noise ratio $(\mathrm{S} / \mathrm{N})$ were 3:1 and 10:1 respectively [22].

\section{Pharmacokinetic and GI transit studies in rats}

Pharmacokinetic studies on AmB, PAR and SSZ and GI transit studies on the SLNs was performed using three male SpragueDawley rats (300-350 g), fasted overnight. The protocol used for this study was approved by the Animal Ethics Committee of the University of Science, Malaysia. The animals were obtained from the Animal Holding Unit of University of Science, Malaysia.

Drug concentrations for AmB, PAR and SP in plasma were obtained after oral administration of drug-loaded SLNs to the rats. The three SLNs (AmB, PAR and SSZ) were similarly formulated and characterized physicochemically as regards to similarity in particle size, surface charge, morphology and in-vitro release properties in our previous work [8] prior to the in-vivo study. SP absorption into plasma was preceded by cleaving of SSZ in the colon by colonic bacteria to produce SP and 5-aminosalicylic acid. Each dose contained an equivalent of $10 \mathrm{mg} / \mathrm{kg}$ of the three individual SLNs dispersed in $1 \mathrm{~mL}$ of distilled water and then administered by oral gavages to the rats.

Blood samples $(300 \mu \mathrm{L})$ were collected from the tail of the rats at $0,1,2,4,8,12$ and $24 \mathrm{hr}$ post-administration into heparinized microcentrifuge tubes. The plasma was immediately harvested from the blood samples by centrifugation and analyzed for drug content using the above HPLC method.

\section{Statistical analysis}

The data have been presented as mean \pm standard deviation (SD) where indicated. GraphPad Prism 5 software was used in the statistical analyses and also to obtain the pharmacokinetic data presented.

\section{Results and Discussion}

\section{Validation of the method}

A number of HPLC methods specific to the detection of only AmB and suitable internal standards in biological fluids have been reported [23-27]. In the present study, we propose a method for the simultaneous analysis of AmB as well as PAR, SP and the IS, suitable for the indirect GI transit monitoring of AmB. Piroxicam was employed as the IS in the present study because it was found to absorb significantly at the two detection wavelengths used (254 and $405 \mathrm{~nm}$ ). The retention times for PAR, SP, AmB and the IS were 8.5, 9.9, 11.7 and $12.8 \mathrm{~min}$ respectively. No interfering peaks were observed at the retention times for all four drugs in blank rat plasma as shown in Figures 1(a) and (b), indicating that the method is specific for the drugs. Furthermore, the peaks obtained for the respective drugs were well-resolved and separate from each other. The baselines are indicative of minimal noise interference (Figures 2 and 3). 

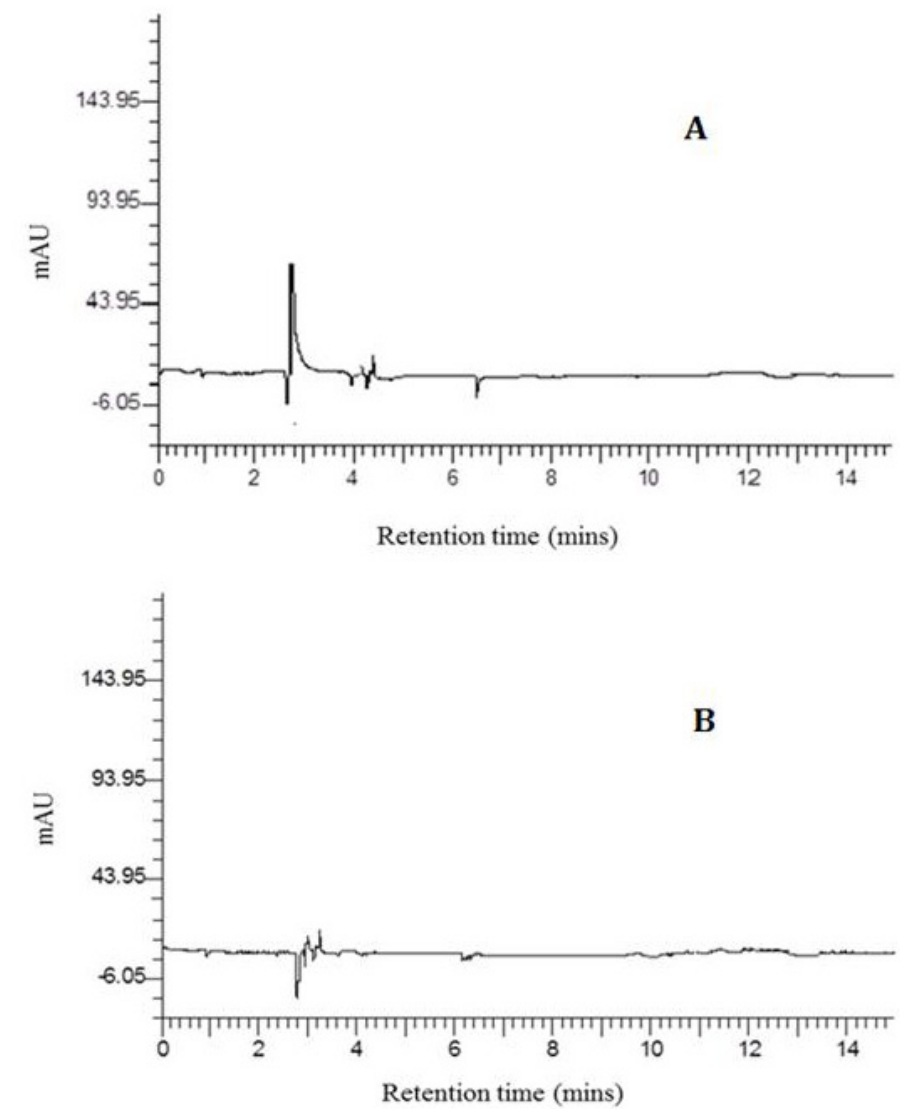

Figure 1: Chromatogram of blank plasma (a) at $254 \mathrm{~nm}$ and (b) at $405 \mathrm{~nm}$

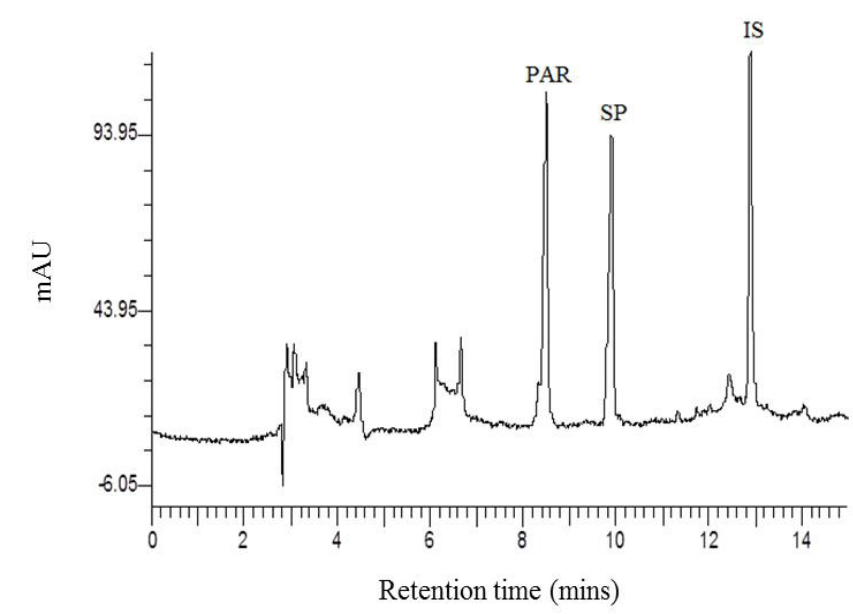

Figure 2: Chromatogram of plasma spiked with PAR, SP and IS at $254 \mathrm{~nm}$

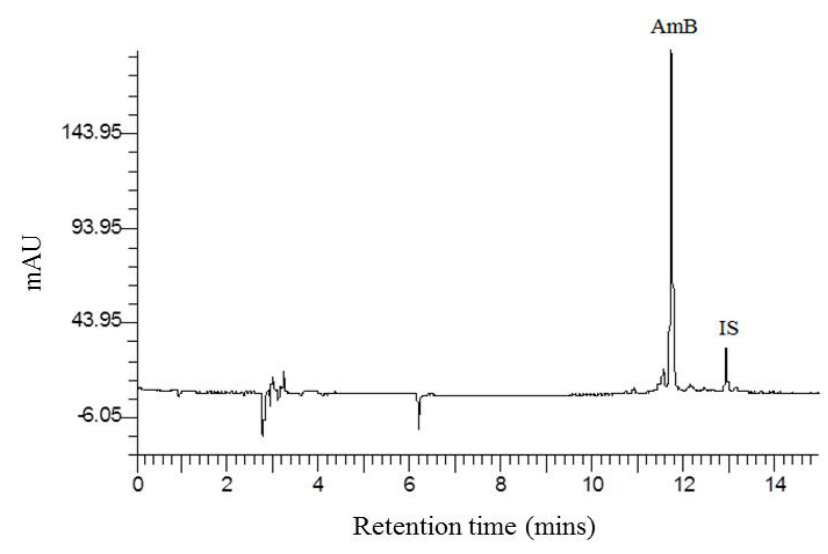

Figure 3: Chromatogram of plasma spiked with AmB and IS at $405 \mathrm{~nm}$ 
The calibration curves for AmB, PAR and SP in plasma, were found to be linear over the concentration ranges of 0.05 - $10 \mu \mathrm{g} / \mathrm{mL}$; $0.5-100 \mu \mathrm{g} / \mathrm{mL}$ and $0.1-20 \mu \mathrm{g} / \mathrm{mL}$ respectively. The coefficients of determination $\left(\mathrm{R}^{2}\right)$ were found to be 0.9999 for AmB; 0.9990 for PAR; and 0.9999 for SP.

The recovery of each analyte from plasma at the concentrations studied is shown in Table 1, pointing to a good drug recovery of the method with a minimum value of $91.70 \%$ ( $<9 \%$ matrix affect).

\begin{tabular}{|c|c|c|c|}
\hline \multirow{2}{*}{ Level } & \multicolumn{3}{|c|}{ \% Recovery $($ mean \pm SD; $n=6)$} \\
\cline { 2 - 4 } & AmB & PAR & SP \\
\hline LC & $97.8 \pm 3.15$ & $98.7 \pm 1.53$ & $97.5 \pm 5.42$ \\
\hline MC & $95.6 \pm 6.12$ & $100.1 \pm 5.33$ & $99.2 \pm 4.76$ \\
\hline HC & $92.1 \pm 0.58$ & $99.4 \pm 1.34$ & $91.7 \pm 1.82$ \\
\hline
\end{tabular}

Table 1: Recovery values for AmB, PAR and SP in rat plasma

The percent accuracies, which estimates the closeness of the observed concentrations to the expected values are shown in Table 2 , with the maximum error obtained being $7.50 \%$. The precision of the method, expressed as \% coefficient of variation was assessed in terms of intra-day and inter-day variability's and the values are expressed in Table 3.

\begin{tabular}{|c|c|c|c|c|c|c|}
\hline \multirow{2}{*}{ Level } & \multicolumn{6}{|c|}{ \% Accuracy (\% Relative error) $(n=6)$} \\
\cline { 2 - 7 } & \multicolumn{2}{|c|}{ AmB } & \multicolumn{2}{c|}{ PAR } & \multicolumn{2}{c|}{ SP } \\
\hline LC & 2.40 & 0.27 & 0.90 & 5.06 & 1.55 & 1.29 \\
\hline MC & 3.20 & 3.90 & 1.44 & 1.81 & 1.60 & 7.50 \\
\hline HC & 0.20 & 1.20 & 0.27 & 2.90 & 0.08 & 1.67 \\
\hline
\end{tabular}

Table 2: Intra-day and inter-day accuracy of the HPLC method

\begin{tabular}{|c|c|c|c|c|c|c|}
\hline \multirow{3}{*}{ Level } & \multicolumn{6}{|c|}{$\%$ Precision $(\% \mathrm{CV})(n=6)$} \\
\hline & \multicolumn{2}{|c|}{ AmB } & \multicolumn{2}{|c|}{ PAR } & \multicolumn{2}{|c|}{ SP } \\
\hline & Intra-day & Inter- day & Intra-day & Inter- day & Intra-day & Inter- day \\
\hline LC & 4.39 & 9.41 & 2.53 & 6.08 & 7.31 & 1.13 \\
\hline MC & 7.17 & 2.36 & 4.01 & 2.87 & 9.89 & 4.79 \\
\hline $\mathrm{HC}$ & 5.78 & 0.93 & 4.28 & 5.13 & 3.77 & 3.16 \\
\hline
\end{tabular}

Table 3: Intra-day and inter-day precision of the HPLC method

The $\% \mathrm{CVs}$ were found to be less than $10 \%$ in all cases, indicating an acceptable degree of variation at the respective concentrations.

The limits of detection (LOD) and quantification (LOQ) were respectively, $5.7 \mathrm{ng} / \mathrm{mL}$ and $19 \mathrm{ng} / \mathrm{mL}$ for AmB; $13.0 \mathrm{ng} / \mathrm{mL}$ and 44.8 $\mathrm{ng} / \mathrm{mL}$ for PAR; and $15.1 \mathrm{ng} / \mathrm{mL}$ and $51.0 \mathrm{ng} / \mathrm{mL}$ for SP. The LOD and LOQ values for AmB and PAR were found to be comparable with or lower than those from published data [23-28] in which similar mobile phase systems were used for AmB. In the present study, all four drugs were analyzed in a two-step procedure, thus minimizing analysis time. Furthermore, this HPLC method has the advantage of a simple sample preparation procedure as compared to solid-phase extraction [25] and also has a relatively short run time of $15 \mathrm{~min}$, considering that four different compounds (including the IS) can be simultaneously analyzed.

\section{Pharmacokinetic and GI transit study}

In order to test the viability of the method, a pilot pharmacokinetic and GI study was conducted using three male Sprague-Dawley rats. The three SLNs exhibited identical physicochemical characteristics [8]. In-vitro release in phosphate buffered saline (pH 7.4) over an $8 \mathrm{hr}$ period showed a potential of prolonged drug release for AmB (11.8\%) and SSZ (31.4\%) in contrast to a faster release for PAR (65.9\%) from their respective SLNs [8]. Figure 4 and Table 4 show the plasma drug concentration-time profiles and pharmacokinetic data respectively, for AmB, PAR and SP following simultaneous oral administration of AmB, PAR and SSZ SLNs to the rats. $\mathrm{A} \mathrm{T}_{\max }$ of about $1 \mathrm{hr}$ was observed from PAR, indicating that it was rapidly released from the PAR SLN due to its preferred solubility in the surrounding aqueous medium as compared with AmB and SSZ which are more hydrophobic. The rapidly released and absorbed PAR in the small intestine therefore estimates the gastric emptying time of the SLNs. AmB was slowly absorbed from the GI tract, reaching a $\mathrm{T}_{\max }$ in $8 \mathrm{hr}$. This is indicative of a slow uptake process most likely via the Peyer's patches, which are located mainly in the ileum, prior to drug emptying into systemic circulation [11]. There was a lag time of about $2 \mathrm{hr}$ prior to appearance of SP in the plasma, which estimates the cecal arrival time of the SLNs. The delay in the absorption of SP is due to a combination of delayed microbial degradation of SSZ in the colon by microbial flora to produce SP and 5- amino salicylic acid followed by its absorption. 


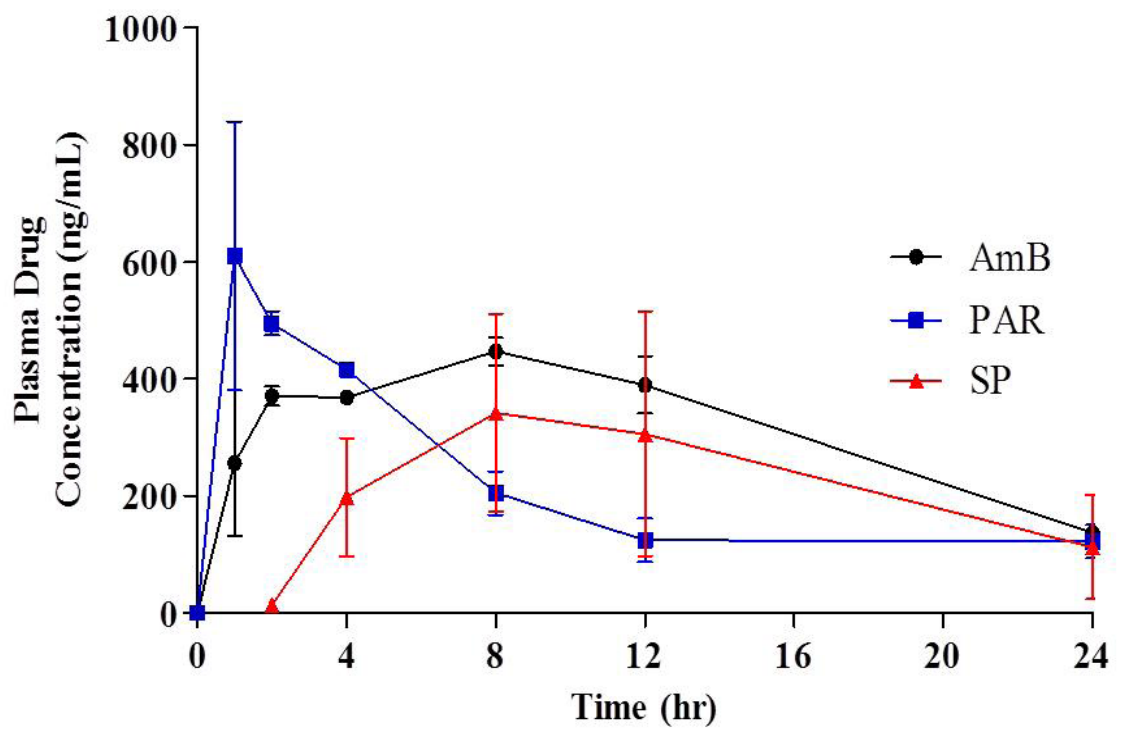

Figure 4: Mean plasma concentration-time profiles of AmB, PAR and SP after a single simultaneous oral gavage of AmB, PAR and SSZ SLNs (10 mg/kg each) simultaneously (mean $\pm \mathrm{SD} ; \mathrm{n}=3$ )

\begin{tabular}{|c|c|c|c|c|}
\hline Drug & $\begin{array}{c}\text { Dose } \\
(\mathbf{m g} / \mathbf{k g})\end{array}$ & $\begin{array}{c}\mathrm{T}_{\max } \\
(\mathbf{h r})\end{array}$ & $\begin{array}{c}\mathbf{C}_{\max } \\
(\mathbf{n g} / \mathbf{m L})\end{array}$ & $\begin{array}{c}\mathbf{A U C}_{\mathbf{0}-24} \\
(\mathbf{n g} \cdot \mathbf{h r} / \mathbf{m L})\end{array}$ \\
\hline AmB & 10 & 8 & $446.7 \pm 23.6$ & $7638.4 \pm 440.3$ \\
\hline PAR & 10 & 1 & $609.8 \pm 229.5$ & $5146.2 \pm 356.2$ \\
\hline SP (from SSZ) & 10 & 8 & $341.7 \pm 169.1$ & $5095.3 \pm 3186.2$ \\
\hline
\end{tabular}

Table 4: Pharmacokinetic data of AmB, PAR and SP following simultaneous oral administration of AmB, PAR and SSZ SLNs $(n=3)$

The $\mathrm{AUC}_{0-24}$ was significantly high for AmB compared to PAR and SP, indicating a favorable albeit slow, uptake process. The high magnitude of $\mathrm{C}_{\max }$ for PAR is indicative of a rapid rate of absorption from the small intestine which is likely to be more as a result of released PAR than due to uptake of the SLNs by Peyer's patches followed by emptying. The pharmacokinetic data obtained is comparable with those reported for lipid based AmB formulations [10,29,30]. We may conclude that the developed HPLC method is suitable for analyzing AmB, PAR and SP in rat plasma and may therefore be employed in pharmacokinetic studies on these drugs and in the indirect estimation of GI transit properties of dosage forms.

\section{Conclusion}

The above analytical method for the simultaneous detection of AmB, PAR and SP is rapid, accurate and sensitive for determining the concentrations of these drugs in plasma, using piroxicam as the IS. The peaks for all the analytes are well resolved and the run time is considerably short, given that four drugs were simultaneously analyzed. The sample volume is small, which is crucial during pharmacokinetic studies on rodents. Crucially, the method can potentially be applied to studying the pharmacokinetics and the GI transit properties of $\mathrm{AmB}$ as well as other dosage forms by the indirect approach.

\section{Acknowledgement}

This work was supported by the Ministry of Science, Technology and Innovation (MOSTI) [02-02-12-SF0227].

\section{References}

1. Wong-Beringer A, Jacobs RA, Guglielmo BJ (1998) Lipid formulations of amphotericin B: Clinical efficacy and toxicities. Clin Infect Dis 27: 603-18.

2. Kleinberg M (2006) What is the current and future status of conventional amphotericin B? Int J Antimicrob Agents 27: 12-6.

3. Patel R (2000) Amphotericin B colloidal dispersion. Expert Opin Pharmacother 1: 475-88.

4. Chia J, McManus E (1990) In-vitro tumor necrosis factor induction assay for analysis of febrile toxicity associated with amphotericin B preparations. Antimicrob Agents Chemother 34: 906-8.

5. Arning M, Kliche KO, Heer-Sonderhoff AH, Wehmeier A (1995) Infusion related toxicity of three different amphotericin B formulations and its relation to cytokine plasma levels. Mycoses 38: 459-65.

6. Tan SW, Billa N, Roberts CR, Burley JC (2010) Surfactant affects on the physical characteristics of amphotericin B-containing nanostructuredlipid carriers. Colloids Surf A 372: 73-9.

7. Tan SW, Billa N (2014) Lipid effects on expulsion rate of amphotericinB from solid lipid nanoparticles. AAPS PharmSciTech 15: $287-95$. 
8. Amekyeh H, Billa N, Yuen KH, Chin SL (2015) A gastrointestinal transit study on amphotericin B-loaded solid lipid nanoparticles in rats. AAPS PharmSciTech doi:10.1208/s12249-014-0279-4.

9. Gupta S, Dube A, Vyas SP (2013) Development and characterization of amphotericin B loaded solid lipid nanoparticles against experimental visceral leishmaniasis. Pharm Nanotechnol 1: 54-67.

10. Patel PA, Patravale VB (2011) AmbiOnp: Solid lipid nanoparticles of amphotericin b for oral administration. J Biomed Nanotechnol 7: 632-9.

11. Li H, Zhao X, Ma Y, Zhai G, Li L, et al. (2009) Enhancement of gastrointestinal absorption of quercetin by solid lipid nanoparticles. J Control Release 133: 238-44.

12. Ritschel WA, Ritter SM, Beiser CR (1978) Are suppository bases absorbed? Study on peroral and rectal absorption of lipids in rabbits. Pharm Ind 40: 82-8.

13. Tarr BD, Yalkowsky SH (1989) Enhanced intestinal absorption of cyclosporine in rats through the reduction of emulsion droplet size. Pharm Res 6: 40-3.

14. Billa N, Yuen KH, Khader MA, Omar A (2000) Gamma-scintigraphic study of the gastrointestinal transit and in vivo dissolution of a controlled release diclofenac sodium formulation in xanthan gum matrices. Int J Pharm 201: 109-20.

15. Schwarz R, Kaspar A, Seelig J, Künnecke B (2002) Gastrointestinal transit times in mice and humans measured with 27Al and 19F nuclear magnetic resonance. Magnet Reson Med 48: 255-61.

16. Peh KK, Yuen KH (1996) Indirect gastrointestinal transit monitoring and absorption of theophylline. Int J Pharm 139: 95-103.

17. Rahman NU, Yuen KH, Woei WJ (2005) Gastrointestinal transit monitoring and absorption of controlled-release pellets of diltiazem. Pharm Dev Technol 10: 371-9.

18. Sjodin L, Visser S, Al-Saffar A (2011) Using pharmacokinetic modeling to determine the effect of drug and Food on gastrointestinal transit in dogs. J Pharmacol Toxicol Methods 64: 42-52.

19. Heading RC, Nimmo J, Prescott LF, Tothill P (1973) The dependence of paracetamol absorption on the rate of gastric emptying. Br J Pharmacol 47: 415-21.

20. Kellow JE, Borody TJ, Phillips SF, Haddad AC, Brown ML, et al. (1986) Sulfapyridine appearance in plasma after salicylazosulfapyridine. Gastroenterology 91: 396-400

21. Staniforth DH, Coates P, Clarke JGN (1987) An HPLC assay for sulfapyridine in plasma and its use to assess small bowel transit time after the administration of sulfasalazine. Int J Clin Pharmacol Ther Toxicol 25: 406-9.

22. Shabir GA (2004) A practical approach to validation of HPLC methods under current good manufacturing practices. JVT 10: 210-8.

23. Chakrabarty US, Pal TK (2011) Rapid and sensitive high performance liquid chromatography method for the determination of amphotericin b in rat plasma. J Pharm Res 4: 3194-7.

24. Echevarría I, Barturen C, Renedo MJ, Dios-Viéitez MC (1998) High-performance liquid chromatographic determination of amphotericin B in plasma and tissue. Application to pharmacokinetic and tissue distribution studies in rats. J Chromatogr A 819: 171-6.

25. Eldem T, Arican-Cellat N (2001) Determination of amphotericin B in human plasma using solid-phase extraction and high-performance liquid chromatography. J Pharm Biomed Anal 25: 53-64.

26. Italia JL, Singh D, Ravi Kumar MN (2009) High-performance liquid chromatographic analysis of amphotericin B in rat plasma using alpha-naphthol as an internal standard. Anal Chim Acta 634: 110-4.

27. Lambros MP, Abbas SA, Bourne DWA (1996) New high-performance liquid chromatographic method for amphotericin B analysis using an internal standard. J Chromatogr B 685: 135-40.

28. Abu-Qare AW, Abou-Donia MB (2001) A validated HPLC method for the determination of pyridostigmine bromide, acetaminophen, acetylsalicylic acid and caffeine in rat plasma and urine. J Pharm Biomed Anal 26: 939-47.

29. Jain S, Valvi PU, Swarnakar NK, Thanki K (2012) Gelatin coated hybrid lipid nanoparticles for oral delivery of amphotericin B. Mol Pharm 9: $2542-53$.

30. Risovic V, Boyd M, Choo E, Wasan KM (2003) Effects of lipid-based oral formulations on plasma and tissue amphotericin b concentrations and renal toxicity in male rats. Antimicrob Agents Chemother 47: 3339-42. 